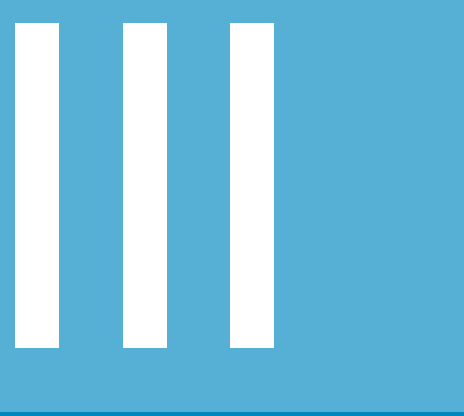

\title{
MISIÓN EDUCACIONAL DE LA UNIVERSIDAD PERUANA UNIÓN, SEDE TARAPOTO,Y SU PERCEPCIÓN EN PÚBLICOS SELECTOS
}

\section{The Educational Mission of the Peruana Union University Head- quarters located in Tarapoto and Perception in Selected Public}

\section{Josué Turpo}

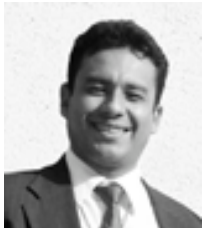

Licenciado en Teología por la Universidad Peruana Unión. Doctorando en Educación por la Universidad Peruana Unión. Actualmente se desempeña como subdirector de investigación de la Universidad Peruana Unión y docente de la cátedra de investigación de la Facultad de Ciencias Empresariales. 


\section{Resumen}

Esta investigación busca conocer la percepción de si la Universidad Peruana Unión cumple o no, lo que dice en su Declaratoria de Misión a través del análisis FODA (fortalezas, oportunidades, debilidades y amenazas). Se obtuvo una muestra de 121 personas distribuidas entre alumnos universitarios, profesores, promotora, administración y ex alumnos. Se aplicó un cuestionario FODA para obtener información de la Universidad Peruana Unión con relación a su Declaratoria de Misión. Los resultados de estas pruebas fueron analizados a través del paquete de análisis estadístico para la investigación educativa SPSS (15.0), mediante el análisis de frecuencias. Entre los resultados se comprobó que la Universidad Peruana Unión muestra una percepción positiva en relación al cumplimiento de su misión por parte de los públicos estudiados. El análisis mostró el ambiente estudiantil como la principal fortaleza con $61.2 \%$, revelando que es una de las áreas fuertes de la UPeU, Tarapoto.

Palabras claves: Percepción, misión, fortalezas, debilidades, amenazas, oportunidades.

\section{Abstract}

This research seeks to understand the perception of whether the Peruana Union University meets or not, with what it says in his declaration of mission through a SWOT (Strengths, Weaknesses, Opportunities and Threats). A sample of 121 people distributed among university students, teachers, developer, administration, and ex-student was made. Also, a questionnaire was administered to obtain information about SWOT (Strengths, weaknesses, opportunities and threats) of the Peruana Union University in relation to their declaration in their Mission. The results of these tests were analyzed through statistical analysis package SPSS for educational research (15.0), by frequency analysis. The results found that the Peruana Union University shows a positive perception in relation to fulfill its mission regarding to the studied population. The analysis showed that student's environment was as their main fortress with $61.2 \%$ showing that it is one of the main areas of UPeU, Tarapoto.

Keywords: Perception, mission, strengths, weaknesses, threats, opportunities 


\section{INTRODUCCIÓN}

La Universidad Peruana Unión (en adelante UPeU) tiene veintiocho años de existencia reconocida como institución educativa de la Iglesia Adventista. Se inició en el año 1919 denominándose Instituto Industrial, pasando por nombres como Colegio Unión y Centro de Educación Superior Unión y Universidad Unión Incaica, llegando al nombre actual de Universidad Peruana Unión.

El 2001, la UPeU de acuerdo con su política expansiva, crea la filial Juliaca y el 2006, la filial en la ciudad de Tarapoto, departamento de San Martín. La Filial de la UPeU Tarapoto, se encuentra situada en el distrito de Morales, provincia de Tarapoto, región San Martín. Es accesible por carretera y avión. Cuenta con más de 750 alumnos y brinda 6 carreras universitarias.

La misión de la UPeU declara que: "La Universidad Peruana Unión es una institución educativa de la Iglesia Adventista del Séptimo Día que forma integralmente profesionales e investigadores competentes y creativos, capaces de brindar un servicio cristiano a la Iglesia y sociedad para restaurar en el ser humano la imagen de Dios".

La importancia de conocer si la Universidad Peruana Unión cumple o no lo que dice en su Declaratoria de Misión, es uno de los objetivos de este trabajo. En palabras de Abarca (2009) “Misión y Visión actualmente se constituyen en los pilares sobre los cuales las modernas compañías basan y conforman su estrategia empresarial, delimitan sus metas y planean sus objetivos a corto, mediano y largo plazo" (p. 42).

La misión es considerada por Kotler y Armstrong (2003), como "un importante elemento de la planificación estratégica" porque es a partir de ésta que se formulan objetivos detallados y que son los que guiarán a la empresa u organización. Asimismo, Ferrel y Hirt (2004) comentan que la misión de una organización "es su propósito general".

El objetivo del trabajo de investigación es determinar a través del análisis FODA (fortalezas, oportunidades, debilidades y amenazas) la percepción de los públicos selectos (promotora, alumnos, profesores, ex alumnos) en relación a la misión de la UPeU.

En el análisis FODA se utiliza la técnica de Kotler (et al:1995), que enfoca el "análisis de los factores claves" para el éxito de su negocio y el análisis de los factores externos y luego el análisis de los factores internos a la institución.

La parte interna tiene que ver con las fortalezas y las debilidades de su institución, aspectos sobre los cuales se tiene algún grado de control. 
La parte externa mira las oportunidades que ofrece, el mercado y las amenazas que debe enfrentar la institución en el mercado seleccionado.

Un modelo de aplicación de este sistema puede verse en el trabajo de David (1997), donde se esquematiza los factores claves y factores estratégicos. Asimismo, Murillo (2005), realizó estudios similares con este análisis.

\section{Fortalezas y debilidades}

Se considera áreas como las siguientes: análisis de recursos, de capital, humanos, sistemas de información, activos fijos, activos no tangibles. También análisis de actividades: recursos gerenciales estratégicos, creatividad. Así como análisis de riesgos y análisis de portafolio.

\section{Oportunidades y amenazas}

Las oportunidades organizacionales se presentan en aquellas áreas que podrían generar muy altos desempeños. Las amenazas organizacionales están en aquellas áreas donde la empresa encuentra dificultades para alcanzar altos niveles de desempeño: se considera el análisis externo, de grupos, de interés, etc.

\section{Materiales y métodos}

El ti de estudio es no-experimental, ya que es descripti a, sin exposición de un estí a las personas encuestadas. El ambiente en el cual se desarrolló la investi ación es la fi de la Universidad Peruana Unión, que se ubica en la ciudad de Tarapoto, en el departamento de San Martí Los grupos que están involucrados son: 85 alumnos. 6 graduandos, 6 administradores, 19 docentes y 5 administradores de la promotora, haciendo un total de 121 personas.

Para la recolección de datos se siguieron los siguientes pasos:

a. Procedimiento de recolección de datos. Se solicitó a la administración de la Universidad Peruana Unión la autorización para desarrollar la investigación en la UPeU, FT.

b. Se obtuvo el cuestionario al estilo FODA. Una vez revisado, se tomó una muestra piloto, aplicando este cuestionario a 25 alumnos para detectar si entienden y contestan las preguntas. Una vez validado el instrumento se procedió a encuestar a los grupos seleccionados. 
c. Reunión formal con la administración y los alumnos para informar los alcances del trabajo de investigación y el beneficio de la encuesta. Se usó el análisis de frecuencias y se utilizó el paquete estadístico SPSS 15.0 en español.

Para encontrar la percepción de los públicos selectos sobre el cumplimiento de la misión, se utilizó el instrumento análisis FODA para colectar un conjunto de información relevante, proveer un análisis de los datos recogidos y desarrollar una propuesta para responder a las debilidades y amenazas que se hayan observado.

El cuestionario FODA cuenta con 45 preguntas, distribuidas de la siguiente manera: 10 sobre identidad adventista, 14 sobre formación integral, 7 sobre investigación, 6 sobre servicio cristiano a la iglesia y sociedad, 7 preguntas sobre restauración del estudiante a la imagen de Dios, y 1 pregunta abierta sobre los cambios o mejoras para cumplir eficientemente la misión.

Para validar el instrumento se solicitó el juicio de expertos, siendo validado por el ingeniero Effer Apaza, docente y especialista en la cátedra de estadísti a, así como por el magister Esteban Tocto especialista en planifi ación estratégica, quienes validaron el instrumento para el tema de misión. Estos expertos también evaluaron la claridad, concisión y relevancia de los enunciados.

Posteriormente se procedió a la validación estadística mediante el alfa de Cronbach que mide la consistencia interna arrojando un coeficiente total de .930 que es valorado como alto o consistente (Hernández, Fernández, y Baptista; 2003).

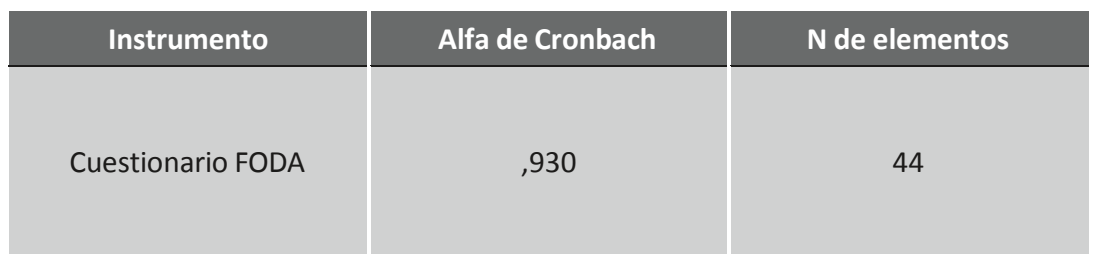

En las preguntas 1-23 se presenta un conjunto de elementos (internos) propios de la institución universitaria, que serán evaluados como fortalezas o debilidades de la misma. Una fortaleza de la institución es aquel factor positivo que le permite destacar entre otras universidades y dar un mejor servicio educativo a la comunidad. Una debilidad de la institución es aquel 
factor negativo que representa un obstáculo o problema que dificulta significativamente que la institución educativa cumpla con su misión.

En las preguntas 24-44 se presenta un conjunto de elementos (externos) del entorno de la institución educativa que serán evaluados como oportunidades o amenazas de la misma. Una oportunidad es aquel factor favorable de los entornos social, económico, político y ecológico, que facilitó el desarrollo de la universidad en el cumplimiento de su misión educativa. Una amenaza es aquel factor negativo del entorno, que impide el desarrollo de la institución y la impartición de un servicio de calidad.

Cada elemento, ya sea interno o externo de la escuela, será evaluado en una escala que tiene cinco posibles opciones: $-2,-1,0+1$ y +2 . El significado de cada dígito es uno de los siguientes:

-2 Esta puntuación significa que al considerar un elemento interno o externo de la universidad, se lo valore como:

a) una debilidad mayor de su escuela o

b) una amenaza mayor para su escuela.

-1 Esta puntuación significa que al considerar un elemento interno o externo de su universidad, se considere si es:

a) una debilidad menor de su escuela o

b) una amenaza menor para su escuela.

0 Esta puntuación significa que al considerar un elemento interno o externo de su universidad, se lo aprecie como que no es:

a) ni una debilidad ni una fortaleza de su escuela o

b) ni una amenaza ni una oportunidad para su escuela.

+1 Esta puntuación significa que al considerar un elemento interno o externo de su universidad, se lo valore como:

a) una fortaleza menor de su escuela o

b) una oportunidad menor para su escuela

+2 Esta puntuación significa que al considerar un elemento interno o externo de su universidad, se considere que es:

a) una fortaleza mayor de su escuela o

b) una oportunidad mayor para su escuela 
Existe además una sexta opción señalada con la expresión na/so. Esto significa "no se aplica/sin opinión" y se incluye para marcar aquellos casos en que el evaluador crea que el reactivo no es aplicable a la situación particular de la universidad que está evaluando o cuando carece de una opinión sobre el reactivo, que le impide evaluarlo de acuerdo con la escala previamente descrita.

Al finalizar el instrumento se presenta una pregunta abierta sobre los cambios o mejoras para que la UPeU cumpla su misión.

Descripción de los grupos encuestados

- Alumnos: Los matriculados como alumnos del primer semestre 2011-I y permanecieron como tales hasta el final del semestre. El total de alumnos es de 750.

- $\quad$ Graduados: Quienes participaron en los actos de graduación el 2010.

- Profesores: Todos los docentes a tiempo completo.

- Administradores: Los tres administradores de la filial y los coordinadores de facultad.

- Promotora: Administradores y departamentales de la Misión Nor Oriental.

\section{RESULTADOS Y DISCUSIÓN}

Tabla 1. Descriptivos de fortalezas

\begin{tabular}{lcc}
\multicolumn{1}{c|}{ Ítem } & $\begin{array}{c}\text { Fortaleza } \\
\text { menor }\end{array}$ & $\begin{array}{c}\text { Fortaleza } \\
\text { mayor }\end{array}$ \\
$\begin{array}{l}\text { 18. El ambiente estudiantil } \\
61.2 \%\end{array}$ & $58 \%$ \\
1. El nivel de dependencia de la UPeU con la promotora, & \\
la Iglesia Adventista & $57 \%$ \\
21. Evaluación de docentes por parte de los alumnos & \\
22. Las instalaciones deportivas & $52.9 \%$ \\
8. El desempeño de los profesores como facilitadores \\
de procesos de aprendizaje integral
\end{tabular}

Fuente: Elaboración propia 
Tabla 2. Descriptivos de debilidades

\begin{tabular}{|c|c|c|}
\hline Ítem & $\begin{array}{l}\text { Debilidad } \\
\text { menor }\end{array}$ & $\begin{array}{l}\text { Debilidad } \\
\text { mayor }\end{array}$ \\
\hline $\begin{array}{l}\text { 13. El acervo bibliográfico en la biblioteca, para formar } \\
\text { investigadores competentes }\end{array}$ & & $31.4 \%$ \\
\hline
\end{tabular}

Fuente: Elaboración propia

Tabla 3. Descriptivos de oportunidades

\begin{tabular}{lc}
\multicolumn{1}{c}{ Ítem } & $\begin{array}{c}\text { Oportunidad } \\
\text { Oportunidad } \\
\text { mayor }\end{array}$ \\
$\begin{array}{l}\text { 36. Apertura de nuevas carreras } \\
\text { 39.El prestigio de la institución }\end{array}$ & $66.9 \%$ \\
44. Convenios con universidades e instituciones & $65.3 \%$ \\
denominacionales y/o foráneas para intercambio & $57.9 \%$ \\
estudianti & \\
43. Énfasis en la educación continua (maestría, & $54.5 \%$ \\
doctorado, diplomados, etc.) & \\
de las políticas educativas de la actual adminis- & \\
tración & \\
cativas que se estén gestando, como resultado & \\
&
\end{tabular}

Fuente: Elaboración propia 


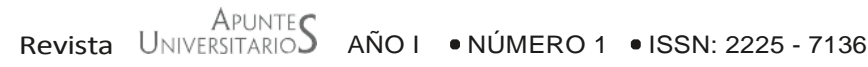

Tabla 4. Descriptivos de amenazas

\begin{tabular}{|c|c|c|}
\hline Ítem & $\begin{array}{l}\text { Amenaza } \\
\text { menor }\end{array}$ & $\begin{array}{l}\text { Amenaza } \\
\text { mayor }\end{array}$ \\
\hline $\begin{array}{l}\text { 40. Ingreso de estudiantes con un nivel de rendimiento } \\
\text { deficiente }\end{array}$ & $37.2 \%$ & \\
\hline $\begin{array}{l}\text { 41. Impacto del humanismo y secularismo en las expe- } \\
\text { riencias de aprendizaje }\end{array}$ & $33.1 \%$ & \\
\hline $\begin{array}{l}\text { 34. Perfil psicológico de las familias de los alumnos de } \\
\text { la institución, que puede afectar en su disposición para } \\
\text { el estudio }\end{array}$ & $25.6 \%$ & \\
\hline 35. Demanda de la educación no presencial & $24 \%$ & \\
\hline
\end{tabular}

Fuente: Elaboración propia

Tabla 5. Descriptivos solo alumnos

\begin{tabular}{|c|c|c|}
\hline & Ítem & Porcentaje \\
\hline Fortaleza & $\begin{array}{l}\text { 1. El nivel de dependencia de la UPeU } \\
\text { con la promotora la Iglesia Adventista }\end{array}$ & $60 \%$ \\
\hline Debilidad & $\begin{array}{l}\text { 13. El acervo bibliográfico en la bi- } \\
\text { blioteca para formar investigadores } \\
\text { competentes }\end{array}$ & $28.2 \%$ \\
\hline Oportunidad & 36. Apertura de nuevas carreras & $69.4 \%$ \\
\hline Amenaza & $\begin{array}{l}\text { 40. Ingreso de estudiantes con un nivel } \\
\text { de rendimiento deficiente }\end{array}$ & $36.5 \%$ \\
\hline
\end{tabular}

Fuente: Elaboración propia 
Tabla 6. Descriptivos solo graduandos

\begin{tabular}{llc} 
Fortaleza & \multicolumn{1}{c}{ Ítem } & Porcentaje \\
Debilidad & $\begin{array}{l}\text { 18. El ambiente estudiantil } \\
\text { 13. El acervo bibliográfico en la bi- } \\
\text { blioteca, para formar investigadores } \\
\text { competentes }\end{array}$ & $100 \%$ \\
Oportunidad & $\begin{array}{l}\text { 42 Relación de los egresados con la } \\
\text { iglesia }\end{array}$ & \\
Amenaza & $\begin{array}{l}\text { 32. Situación económica y financiera } \\
\text { del país en general: política económi- } \\
\end{array}$ & $66.7 \%$ \\
& ca, deudas interna y externa, balanza \\
& comercial, PBI, etc.
\end{tabular}

Fuente: Elaboración propia

Tabla 7. Descriptivos solo administradores

\begin{tabular}{llc} 
Fortaleza & \multicolumn{1}{c}{ 14. Se observa un clima espiritual en el } & Porcentaje \\
& campus universitario & $83.3 \%$ \\
Debilidad & $\begin{array}{l}\text { 13. El acervo bibliográfico en la bi- } \\
\text { blioteca, para formar investigadores } \\
\text { competentes }\end{array}$ & $16.7 \%$ \\
Oportunidad & $\begin{array}{l}\text { 39. El prestigio de la institución } \\
\text { Amenaza }\end{array}$ & $\begin{array}{l}\text { 40. Ingreso de estudiantes con un nivel } \\
\text { de rendimiento deficiente }\end{array}$ \\
&
\end{tabular}

Fuente: Elaboración propia 
Tabla 8. Descriptivos solo profesores

\begin{tabular}{llc} 
Fortaleza & \multicolumn{1}{c}{ Ítem } & Porcentaje \\
& $\begin{array}{l}\text { 15. Se observa en los alumnos que están } \\
\text { siendo restaurados a la imagen de Dios }\end{array}$ & $73.7 \%$ \\
Debilidad & $\begin{array}{l}\text { 13. El acervo bibliográfico en la biblioteca } \\
\text { para formar investigadores competentes }\end{array}$ & $57.9 \%$ \\
Oportunidad & 36. Apertura de nuevas carreras & $73.7 \%$ \\
Amenaza & 40. Ingreso de estudiantes con un nivel de & $42.1 \%$ \\
& rendimiento deficiente &
\end{tabular}

Fuente: Elaboración propia

Tabla 9. Descriptivos solo MNO

\begin{tabular}{llc}
\hline Fortaleza & \multicolumn{1}{c}{$\begin{array}{c}\text { Ítem } \\
\text { 17. La disposición de los graduandos de } \\
\text { brindar un servicio a la iglesia y sociedad }\end{array}$} & $80 \%$ \\
Debilidad & $\begin{array}{l}\text { 6. Consolidación del ejercicio perma- } \\
\text { nente de la investigación, el servicio, la } \\
\text { creatividad y la proyección social }\end{array}$ & $20 \%$ \\
Oportunidad & $\begin{array}{l}\text { 33. El plan de estudios con cursos deno- } \\
\text { minacionales (formación cristiana) }\end{array}$ & $80 \%$ \\
Amenaza & $\begin{array}{l}\text { 28. El aumento de profesores no de- } \\
\text { nominacionales (no estudiaron en una } \\
\text { universidad adventista) }\end{array}$ & $40 \%$ \\
\hline
\end{tabular}

Fuente: Elaboración propia

En el resultado de la tabla 1 encontramos que el nivel de dependencia de la UPeU con la IASD (Iglesia Adventi ta del Sépti día) es considerado como una fortaleza, lo que signifi a que la UPeU se encuentra en un nivel aceptable en relación a su misión; del mismo modo, el desempeño de los profesores como facilitadores del proceso de aprendizaje integral, se 
encuentra como una fortaleza menor. El ítem que obtuvo el más alto nivel es el de ambiente estudianti (61.2\%) lo que signifi aría que la vida social en el campus universitario, fruto de las diferentes acti $s$, es percibido por los públicos estudiados de manera positi a. Del mismo modo, la evaluación semestral que hacen los alumnos a sus profesores es considerada también como una fortaleza por más del 57\%, al igual que las instalaciones deportivas, las mismas que fueron inauguradas el año pasado.

En el aspecto de debilidades, la que más se repite es sobre el acervo bibliográfico del área de biblioteca, que tiene porcentajes altos tanto como debilidad menor como debilidad mayor.

Dentro de las oportunidades es muy bien visto el prestigio de la institución (65.3\%), lo que significa que es una oportunidad para el crecimiento institucional. Así como también la apertura de nuevas carreras (66.9\%).

En el área de las amenazas, el ingreso de estudiantes con un nivel de rendimiento deficiente es visto como la mayor amenaza, lo que significa que en el área de admisión no se está desarrollando el filtro correspondiente, asímismo el impacto del humanismo y secularismo, lo que significaría que la tendencia universitaria en el Perú gira en torno a esas corrientes.

En la tabla 7 se observa que los administradores ven un clima espiritual positivo en la universidad, un aspecto que no es distinguido con la misma claridad por otros grupos encuestados.

En la tabla 9 se observa que la promotora ve como una fortaleza la disposición de los graduandos de brindar un servicio a la iglesia y sociedad, por otro lado se observa como amenaza el aumento de profesores no denominacionales, lo que signifi a que se requiere de más personal denominacional (docentes que hicieron sus estudios en insti s adventi tas).

Sobre las respuestas a la pregunta abierta sobre "¿Qué mejoras o cambios harías para que la UPeU pueda cumplir mejor su misión?, contestaron de la siguiente forma, en primer lugar manifestaron que se debe priorizar la exigencia académica, con docentes más capacitados y que exista un equilibrio entre lo espiritual y lo intelectual. La segunda preocupación fue referente al área espiritual que se viva en un ambiente cristiano, de valores, con docentes y administradores como ejemplos de vida.

La tercera preocupación que manifestaron está en relación a la infraestructura, especialmente en el área de la biblioteca y laboratorios, este asunto, relacionándolo con la investi ación, se deben acatar las nuevas políti as sobre investi ación universitaria. Algunos pocos manifestaron su preocupación sobre las pensiones de enseñanza, así como la necesidad de una Ofi de Planifi ación. 
En cuanto a las estrategias en referencia a las debilidades y amenazas, siendo que el área de biblioteca fue la mayor debilidad, se debe contar con un plan de compra de libros, crear el fondo editorial universitario, y contar con técnicos especializados en el área bibliotecaria. La amenaza más fuerte está expresada en el ingreso de estudiantes con nivel deficiente, por lo que se debe fortalecer el área de admisión, los centros preuniversitarios y lo prerrequisitos académicos básicos, para que un alumno ingrese a la vida universitaria. Esto se puede hacer creando un área académica en admisión.

\section{CONCLUSIONES}

1. La fortaleza que más perciben los grupos estudiados es el ambiente estudianti con $61.2 \%$, mostrando que es una de las áreas fuertes de la UPeU, Tarapoto.

2. La debilidad que más perciben los grupos estudiados, aunque no de manera mayoritaria, es el acervo bibliográfico en la biblioteca para formar investigadores competentes el ambiente estudiantil con 31.4\%, mostrando que es una de las áreas débiles de la UPeU, Tarapoto.

3. La oportunidad que más perciben los grupos estudiados es la apertura de nuevas carreras con $61.9 \%$, mostrando que es una de las áreas que puede ser aprovechada por la UPeU, Tarapoto.

4. La mayor amenaza que perciben los grupos estudiados es el ingreso de estudiantes con un nivel de rendimiento deficiente con $37.2 \%$, mostrando que es una de las áreas que debe ser evaluada por la UPeU, Tarapoto.

5. El Análisis FODA aplicado muestra a la UPeU con una percepción positiva en relación al cumplimiento de su misión, por parte de los públicos estudiados.

6. La exigencia académica y un ambiente cristiano saludable son considerados como factores prioritarios por parte de los públicos estudiados, para el cumplimiento de la Misión.

\section{RECOMENDACIONES}

1. Se recomienda construir un instrumento más efecti o para investi ar las fortalezas, debilidades, oportunidades y amenazas de la UPeU. Esto puede lograrse 
refi el que se uti ó, creando un nuevo instrumento o uti ando otro ya existente.

2. Es conveniente efectuar investigaciones similares en la otra filial de la UPeU. Por lo tanto, es recomendable hacer un estudio más amplio que abarque a los tres campos universitarios, incluido sus programas de PROESAD (Programa de educación semi presencial a distancia).

3. Se recomienda ampliar y profundizar la investi ación aquí expuesta, puesto que el análisis FODA es importante para el desarrollo insti y el éxito académico.

Josué Turpo

Universidad Peruana Unión e-mail: editc2003@hotmail.com

Recibido: 2 de agosto de 2011 Aceptado: 5 de septiembre de 2011

\section{Referencias}

Abarca R. (2005). Plan estratégico. Perú: Universidad Católica de Santa María. Disponibilidad web en: http://www.ucsm.edu.pe/rabarcaf/

David F. (1997). Conceptos de administración estratégica. México, D.F.: Editorial Prentice-Hall Hispanoamericana, S. A.

Ferrell O., Hirt G. (2004). Introducción a los negocios en un mundo cambiante. 4a Ed. USA: McGraw-Hill. ISBN 9701039424.

Kotler P., Armstrong G. (2003). Fundamentos del marketing. 6a Ed. Trad. Roberto Escalona. México D. F.: Pearson Education

Kotler P., Fox Karen. (1995). Strategic Marketing for Educational Institutions. 2a. Ed. USA, New Jersey: Prentice-Hall, Englewood Cliff.

Hernández R., Fernández C., Baptista P. (2003). Metodología de la investigación. México: Mc Graw Hill Interamericana DE, S. A. de C. V.

Murillo A. (2005). Manual del uso de la matriz DOFA. Montemorelos: Universidad de Montemorelos. 